\title{
VEGFR-1 targeted DNAzyme via transcatheter arterial delivery influences tumor vasculature assessed through dynamic contrast-enhanced magnetic resonance imaging
}

\author{
LIQING ZHANG $^{1}$, WEI ZHAO ${ }^{1}$, CHEN LIANG $^{2}$, XIAOPING YI $^{1}$, YIGANG PEI $^{1}$, \\ YITING LIN ${ }^{3}$, JIANG $\mathrm{HE}^{3}$ and WENZHENG $\mathrm{LI}^{1}$ \\ ${ }^{1}$ Department of Radiology, Xiangya Hospital, Central South University; ${ }^{2}$ Department of Interventional Radiology, \\ Hospital of Finance and Commerce; ${ }^{3}$ Center for Molecular Medicine, Xiangya Hospital, \\ Central South University, Changsha, Hunan 410008, P.R. China
}

Received January 31, 2016; Accepted March 9, 2016

DOI: $10.3892 / o r .2016 .4933$

\begin{abstract}
DNAzymes are synthetic single-stranded DNA oligonucleotides that bind and cleave target mRNA in a sequence-specific manner. Although the therapeutic potential has been demonstrated in both preclinical and clinical settings, the efficient delivery and in vivo assessment of the DNAzyme efficacy remain the vital unsolved issue. In the present study, we examined the feasibility of using transcatheter arterial chemoembolization (TACE) strategy to deliver a DNAzyme targeting VEGFR-1 and monitoring its effect on tumor angiogenesis in vivo via dynamic contrast enhanced magnetic resonance imaging (DCE-MRI). In a rabbit liver cancer model (VX2), we showed that the DNAzyme was efficiently delivered into the tumor by TACE. DCE-MRI revealed that the VEGFR1-targeted DNAzyme affected the tumor vasculature through inhibiting VEGFR-1 expression in vivo, which was reflected by a reduction of $\mathrm{K}^{\text {trans }}$ and $\mathrm{K}_{\mathrm{ep}}$, the parameters of tumor microvascular permeability. Our findings offer an efficient strategy of delivery and assessment of the VEGFR-1 DNAzyme, and further demonstrate the feasibility of DNAzyme for cancer therapy.
\end{abstract}

\section{Introduction}

Hepatocellular carcinoma (HCC) was one of the most common malignant tumors in 2012, with incidence and mortality rate of 20.7 and 19.4/100,000, respectively (1). Surgical removal of the tumor and chemotherapy is the first line treatment for HCC. However, chemoresistance is one of major issues in the clinical

Correspondence to: Dr Wenzheng Li, Department of Radiology, Xiangya Hospital, Central South University, Changsha, Hunan 410008, P.R. China

E-mail: wenzheng727@163.com

Key words: hepatocellular carcinoma, VEGFR-1, DNAzyme, transcatheter arterial delivery, DCE-MRI treatment of liver cancer. The application of intervention in the clinical transcatheter arterial chemoembolization (TACE) has been widely used for non-surgical method for advanced liver cancer. Recently, TACE in combination with different therapeutics has been intensively investigated at both preclinical and clinical settings for HCC (2).

Angiogenesis plays a critical role in tumor growth, progression and metastasis. Hypoxia induces the hypoxia inducible factor (HIF) family proteins via degradation of the von Hippel-Lindau (VHL) tumor-suppressor (3), and production of angiogenic factors, such as vascular endothelial growth factor (VEGF), fibroblast growth factor (FGF), platelet-derived, epidermal and placental growth factors, and angiopoietins-1 (4). The two major tyrosine kinase receptors are VEGFR-1 and VEGFR-2. Unlike fibroblast growth factor receptors, both VEGF receptors are selectively expressed on endothelial cells (ECs) (5). Depending on the tumor type, VEGF and its two receptors may function via either the autocrine or paracrine mechanisms in humans (6-8). In comparison to the surrounding normal tissue vasculature, in tumor-associated ECs in a variety of tumors, both VEGFR-1 and VEGFR-2 are upregulated (5). Recently, it has been shown that HCC has robust nuclear and cytoplasmic staining for active, phosphorylated VEGF receptor-1. Autocrine VEGF signaling directly promotes HCC cell proliferation and affects the sorafenib treatment outcome in vitro and in vivo (9).

As the elevated expression of VEGF and its receptors have been closely correlated with tumor vascularity, progression and metastasis, targeting of VEGF/VEGFRs becomes a worthwhile approach to cancer therapy. Several strategies have been explored to inhibit VEGFR activity, which include the use of antisense, ribozymes and DNAzymes against VEGFR-1 or VEGFR-2 to suppress the formation of VEGF-induced new blood vessels (10-12).

DNAzymes are synthetic, single-stranded DNA catalysts that bind to their complementary sequence within a target mRNA by Watson-Crick base pairing and subsequently cleave the mRNA at predetermined phosphodiester linkages (13-15). Numerous studies have demonstrated that specific DNAzymes, targeting tumor-associated genes, exerts anticancer effects, 
which supports the efficiency of specific DNAzymes in downregulation of appropriate genes and highlights DNAzymes as potential therapeutic molecules. However, the major hurdle for application of gene-targeted therapeutics in clinical settings remains how to ensure an efficient in vivo delivery.

Dynamic contrast-enhanced magnetic resonance imaging (DCE-MRI), diffusion-weighted imaging (DWI), perfusion-weighted imaging (PWI) and other imaging methods are used to evaluate the efficacy of treatment (16-18). The dynamic contrast images obtained are used to quantitate parameters which characterize tumor microcirculation. For example, the volume constant for the transfer of CA from the plasma to the extravascular extracellular space (e.g., $\mathrm{K}^{\text {trans }} /$ minute) represents an important parameter, and is primarily related to blood perfusion and microvessel permeability. Over the past 10 years, DCE-MRI and extracted kinetic parameters have been applied to phase I and II clinical trials of anti-angiogenic drugs and vascular disrupting agents, with $\mathrm{K}^{\text {trans }}$ generally recognized as a marker of tumor blood flow and permeability. In addition, $\mathrm{K}^{\text {trans }}$ has been recommended as a primary endpoint for anticancer treatment trials by the US National Cancer Institute (19).

In the present study, we investigated whether it is feasible to utilize TACE in combination with the VEGFR-1-targeted DNAzyme for anti-angiogenesis approach to HCC treatment in tumor-bearing rabbits through interventional operation. DCE-MRI imaging is used to evaluate the efficacy of treatment. The present study showed the effect of VEGFR-1 targeted DNAzymes on tumor vasculature assessed by DCE-MRI.

\section{Materials and methods}

DNAzyme and oligonucleotides. DNAzyme and control oligonucleotides were commercially synthesized (Sangon Biotech, Shanghai, China) with an inverted thymidine at the 3 ' position and purified by high performance liquid chromatography (HPLC). The sequences of DNAzyme and its target were previously described (12) and are shown in Fig. 1A.

Cell culture and transfection. Corneal endothelial cells from rabbit cornea were isolated by trypsin (Gibco) digestion and cultured to assay the effect of targeted VEGFR-1 DNAzymes in the rabbit corneal endothelial cells. The present study was approved by the Animal Care and Use Committee of Central South University. Corneal endothelial cells were cultured with Opti-MEM (Gibco) and seeded into 6-well plates 5x10\% $/$ well one day before transfection. When cells reached 70-80\% confluency, cells were washed with serum-free medium and tranfected VEGFR-1 DNAzymes (DZ) or Ctrl DNAzymes with FuGENE (Promega, Madison, WI, USA). Six hours after transfection, cells were used to assay transfection efficiency. At $24 \mathrm{~h}$ post-transfection, cells were allowed to recover in complete medium overnight and were harvested for realtime PCR. A 5' fluorescein isothiocyanate (FITC)-labeled DNAzyme oligonucleotide was used for assessing transfection efficiency in vivo of the VX2 HCC.

Fluorescence-activated cell sorter (FACS) analysis. The transfected corneal endothelial cells were incubated at $37^{\circ} \mathrm{C}$ for $6 \mathrm{~h}$. The cells were washed with $\mathrm{pH} 7.4$ phosphate-buffered saline (PBS), harvested by treatment with trypsin-EDTA, resuspended in ice-cold PBS and immediately examined using a FACSort flow cytometer (FACS; Merck Millipore, Temecula, CA, USA). After 20,000 total events were acquired/sample, transfection efficiency was defined by assessing cell size and granularity in the forward scatter and side scatter channels. FITC fluorescence in the cell population was expressed as the geometric mean of positive events after subtraction of background fluorescence.

Animal model and DZ treatment. The present study was approved by the Animal Care and Use Committee of Central South University. Sixteen New Zealand white rabbits weighing $2.37-2.70 \mathrm{~kg}(2.54 \pm 0.11 \mathrm{~kg})$ were used (The Third Xiangya Experimental Animal Center, Changsha, China), according to the Third Xiangya Hospital Animal Care and Ethics Committee rules [LLSC (LA) 2013-005]. Briefly, the hind limb donor rabbit was anesthetized with intravenous injection of $30 \mathrm{mg} / \mathrm{kg}$ pentobarbital sodium. The tumor tissues were removed avoiding the necrotic core and cut into $1 \mathrm{~mm}^{3}$. These blocs were stored in sterile saline for implantation in $4^{\circ} \mathrm{C}$. After anesthetized, one bloc was planted into the left midial lobe of the rabbit. The treated rabbit was intramuscular injected 800,000 U penicillin (North China Pharmaceutical Group) for 3 days. All procedures were performed in a sterile condition. The VX2 HCC was incubated 10-14 days. Twelve tumor-carrying rabbits were randomly divided into 4 groups $(\mathrm{n}=3)$ : group A, $10 \mu \mathrm{l}$ Fugene $(\mathrm{F})+250 \mu \mathrm{l}$ Lipiodol $(\mathrm{L})+50 \mu 1$ targeted VEGFR-1 DNAzymes (DZ); group B, $10 \mu 1$ Fugene $+250 \mu 1$ Lipiodol $+50 \mu 1$ control oligonucleotides (Ctrl); group C, $10 \mu 1$ Fugene $+250 \mu 1$ Lipiodol; group D, blank.

DCE-MRI scanning schedule and parameters. All MRI examinations were performed with a 3.0-T MR system (Signa HDxt 3.0T; GE Heathcare, WI, USA). The VX2 HCCs were grown for 10-14 days. At the 10th day after modelling, the first MRI scanning was performed and at the 13th day (before intervention as $0 \mathrm{~h}$ point) for second scanning. The third and fourth scannings were performed at 48 and $96 \mathrm{~h}$ after intervention, respectively. Scanning parameters were: $\mathrm{T} 2$ weighted imaging (T2WI) axial images used fast spin-echo (FSE) sequence, echo time $(\mathrm{TE})=85.1 \mathrm{msec}$, repetition time $(\mathrm{TR})=4,800 \mathrm{msec}$, field of view $(F O V)=15 \mathrm{~mm}$ over a $160 \times 256$ matrix, number of excitations $(\mathrm{NEX})=4$, layer thickness $=3 \mathrm{~mm}$, layer number $=16$, interlayer spacing $=0$. T1W1 axial images used FSE sequence, $\mathrm{TE}=7.9 \mathrm{msec} \mathrm{TR}=520 \mathrm{msec}$, field of view $(\mathrm{FOV})=15 \mathrm{~mm}$ over a 128x128 matrix, number of excitations $(\mathrm{NEX})=2$, layer thickness $=3 \mathrm{~mm}$, layer number $=16$, interlayer spacing $=0$. DCE-MRI used volume acceleration-flexible (LAVA-Flex): $\mathrm{TE}=1.2 \mathrm{msec}, \mathrm{TR}=2.7 \mathrm{msec}$, flip angle $12^{\circ}, \mathrm{FOV}=15 \mathrm{~mm}$ over a $128 \times 128$ matrix, actual layer thickness $=3.2 \mathrm{~mm}$, layer number $=2,000$, time resolution $4 \mathrm{sec}$ with 50 time phase, total imaging time $8 \mathrm{~min} 50 \mathrm{sec}$. Scanning steps: 12 New Zealand white rabbits were anesthetized. 22G I.V. catheters were fixed on the left or right ear with a piece of hard paper. The rabbits lay supine placed onto a board, put in the eight-channel knee coil and surrounded by sponge aiming at reducing breathing movement. The advanced mode of scanning was feet first. 
Before scan, we connected 22G I.V. catheters to the high-pressure injectors with disposable intravenous infusion needle (21GX3/4, 0.8x19 mm). On the first time phase, gadopentetate dimeglumine (Gd-BOPTA; Bracco, Milan, Italy), a blood-pool contrast agent, was injected via the tail-vein catheter, and the doses of $0.1 \mathrm{mmol} / \mathrm{kg}, 10 \mathrm{ml}$ saline to flush.

Targeted drug delivery intervention. Twelve tumor-bearing rabbits were randomly divided into 4 groups. Drug admixture consisted of $10 \mu \mathrm{l}$ Fugene and $50 \mu \mathrm{l}$ VEGFR-1 DNAzymes or control. The drug admixture stayed at room temperature (RT) for $8 \mathrm{~min}$, followed by adding $250 \mu \mathrm{l}$ Lipiodol and $10 \mathrm{~min}$ at RT. A 2-3 cm long groin incision was made and blunt dissection down to the femoral bundle of the tumor-bearing rabbits. The femoral artery was separated gently from the accompanying nerve and vein, and punctured with arteriovenous catheter (22Gx1 " 0.9x25 mm) and catheterized with $4 \mathrm{~F}$ sheath and $4 \mathrm{~F}$ catheter. Catheter and micro-catheter was placed near the tumor blood vessels. After digital subtraction angiography (DSA) of the liver, the drug admixture was injected through the micro-catheter followed with $2 \mathrm{ml}$ saline flushing, following pulling out the catheter and ligating femoral artery close to the incision. The treated rabbits were all intramuscular injected with $800,000 \mathrm{U}$ penicillin for 3 days.

In vivo delivery efficiency analysis. Two VX2 tumor-bearing rabbits were used for assessing in vivo delivery efficiency. One was treated with the combination of $5 \mu$ l Fugene, $20 \mu \mathrm{l}$ FITC-labeled DNAzyme and $200 \mu \mathrm{l}$ Lipiodol, the other with the combination of $5 \mu 1$ Fugene, $20 \mu \mathrm{l}$ saline and $200 \mu \mathrm{l}$ Lipiodol. The two rabbits were sacrificed $6 \mathrm{~h}$ after catheter delivery. The VX2 tumor tissue was removed for frozen sections. The $4 \mu \mathrm{m}$ frozen sections was obtained with cryostat microtome (CM3050S) and examined with fluorescence microscope (both from Leica) directly.

DCE-MRI data analysis. These raw data of DCE-MRI were analyzed using NordicICE software (version 2.3.14: NordicNeuroLab, Bergen, Norway) without animal grouping information. The model for contrast agent permeability analysis was based on a two-compartment tissue kinetic model, which obtain quantitative parameters and the reconstruction of parameters map, such as $\mathrm{K}^{\text {trans }}, \mathrm{K}_{\mathrm{ep}}$ and $\mathrm{Ve} \mathrm{K}^{\text {trans }}$ is a rate constant that represents the transfer of contrast agent from the arterial blood into the extravascular extracellular space (EES) (in $\mathrm{ml} / \mathrm{ml} / \mathrm{min}$ ). $\mathrm{K}_{\mathrm{ep}}$ is a rate constant that represents transfer of the contrast agent from the EES to the blood plasma (in/min). Ve is the EES volume of distribution (in milliliters of blood/milliliters of tissue), $\mathrm{Ve}=\mathrm{K}^{\text {trans }} / \mathrm{K}_{\mathrm{ep}}$. All region of interest (ROI) placement were manually performed (avoiding the necrotic core). The ROI areas were $18-25 \mathrm{~mm}^{2}$. We selected the maximum level of tumor and performed two ROIs of each tumor, followed by reconstruction of the maps and the imaging.

Real-time PCR. The RNA was extracted from each tumor tissue with TRIzol reagent (Life Technologies, Carlsbad, CA, USA) and reverse transcribed into cDNA with a reverse transcription kit (Thermo). Real-time PCR was performed by primers for VEGFR-1, 5'-TAGCATCACAAGGGCAGCT
A

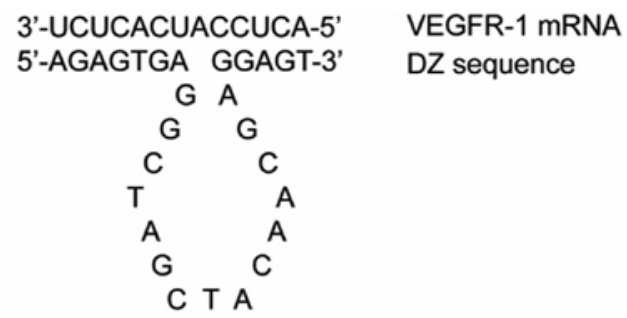

B

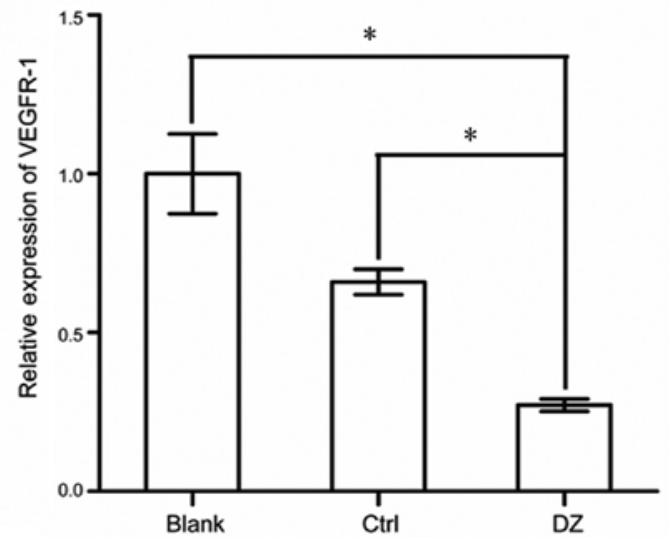

Figure 1. VEGFR-1-targetd DNAzyme and its effect on VEGFR-1 expression in rabbit cells. (A) Design of DNAzymes targeted to human VEGFR-1 mRNA. (B) Expression of VEGFR-1 in the rabbit corneal endothelial cells after transfection of the DNAzyme $\left({ }^{*} \mathrm{P}<0.05\right)$.

TTA-3' (sense) and 5'-CATCTACTATCTTGCACTAAGCC TCT-3' (antisense); GAPDH 5'-CATCATCCCTGCCTCCA CT-3' (sense), 5'-GCCTGCTTCACCACCTTCTT-3' (antisense). The amplification and dissolution curve of the real-time quantitative PCR (Bio-Rad, Hercules, CA, USA) was confirmed after the reaction.

Statistical analysis. Data are presented as mean \pm SD and were analyzed by t-test. Paired comparisons between multiple samples were tested by the Dunnett's test, t-test and Pearson's correlation analysis. All analysis was performed with 17.0 SPSS. A P-value of $<0.05$ was considered to indicate a statistically significant result.

\section{Results}

Downregulation of VEGFR-1 expression in rabbit corneal endothelial cells. VEGFRs, which belong to receptor tyrosine kinase family, play important roles in angiogenesis. We previously showed that VEGFR-1-targeted DNAzymes could suppress angiogenesis by inhibiting VEGFR-1 expression in human endothelial cells. In the present study, we investigated whether the DNAzyme could be delivered into liver and impact on tumor vasculature via a catheter that simulates TACE in rabbit hepatocyte carcinoma. To verify whether the DNAzyme could inhibit rabbit VEGFR-1 expression, the rabbit corneal endothelial cells were cultured and transfected with VEGFR-1-targeted DNAzyme using Fugene. The results showed that a marked decrease of VEGFR-1 expression was observed in the cells treated with VEGFR DNAzyme compared with control DNAzyme. While only a marginal decrease in mRNA level was observed in the 

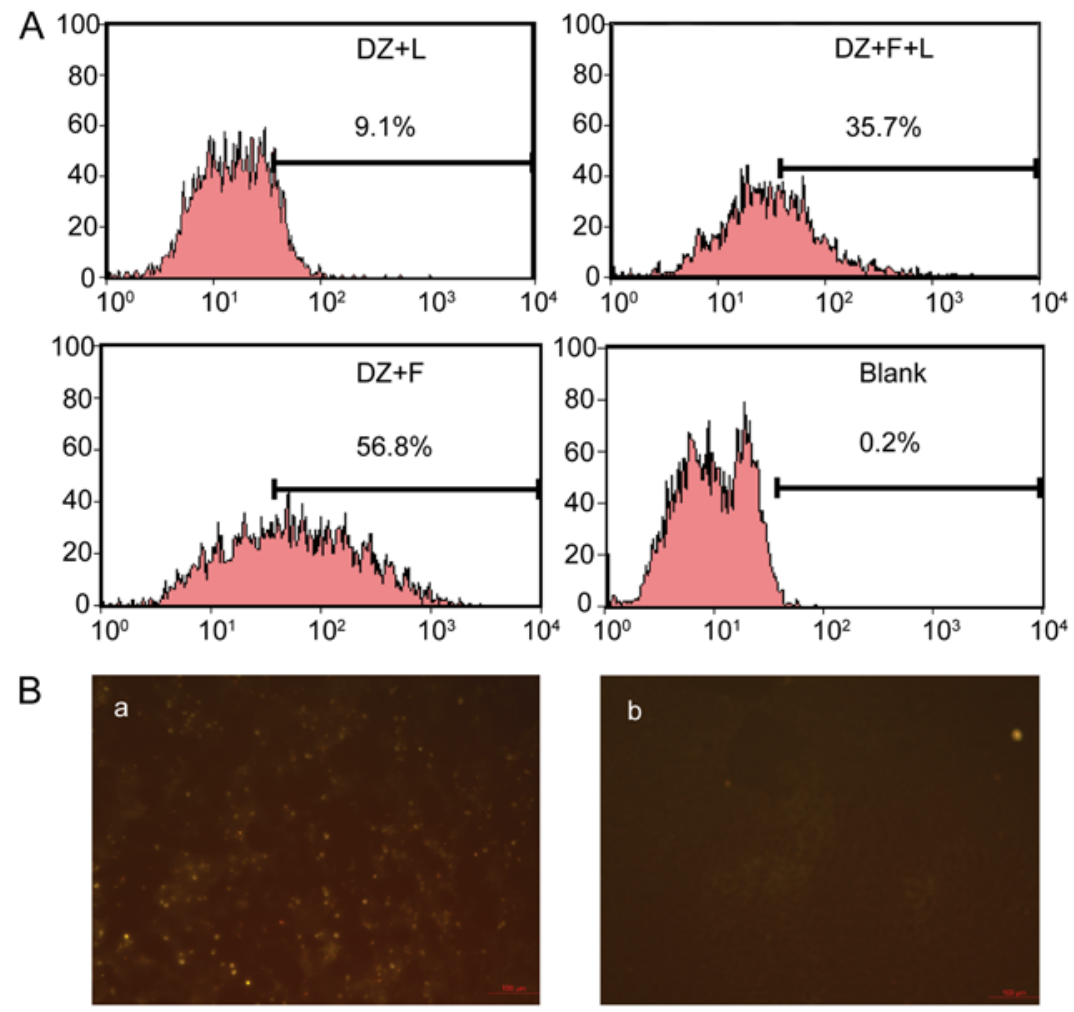

Figure 2. Assessment of the transfection efficiency of VEGFR-1 DNAzymes mixed with Lipiodol. (A) In vitro analysis of rabbit corneal endothelial cells by FACS. (B) In vivo analysis of liver tissues in tumor-bearing rabbits: a, FITC-labeled DNAzymes + Fugene + Lipiodol; b, non-labeled control + saline + Fugene + Lipiodol.

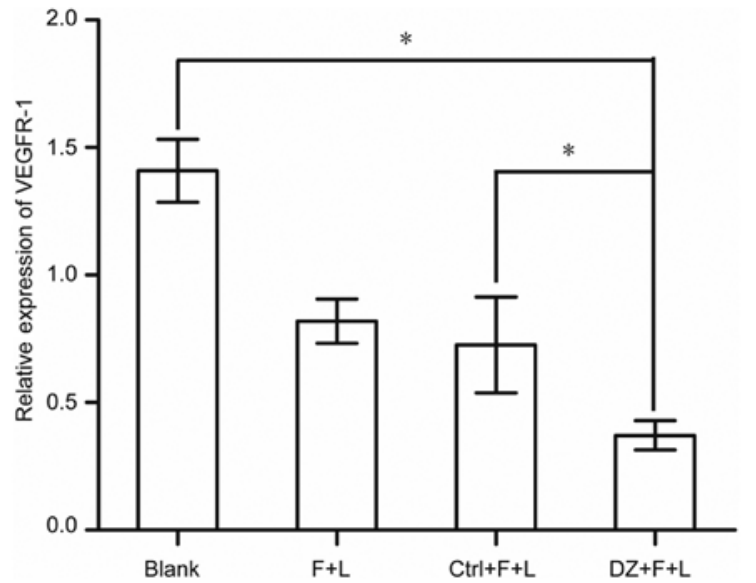

Figure 3. Expression of VEGFR-1 mRNA in the tumor tissues. The animals were sacrificed 8 days after treatment and qRT-PCR was performed. ${ }^{*} \mathrm{P}<0.05$ compared with Ctrl group ( $\mathrm{n}=3)$.

cells treated with control DNAzyme compared with blank, which may result from an antisense effect of the DNAzyme binding arms (Fig. 1B). Thus, these results demonstrated that VEGFR-1 DNAzyme is effective in downregulation of VEGFR-1 of rabbit origin.

Cellular uptake efficiency of Lipiodol-Fugene/DZ in rabbit corneal endothelial cells. Lipiodol can embolize the blood vessels and make the anticancer drugs selectively retainable at a high concentration in tumor tissues for a long time. To determine whether VEGFR-1 DNAzyme could be released from the Lipiodol-Fugene/DZ complex, DZ was labeled with FITC at the 5 ' end. Rabbit corneal endothelial cells were transfected with the complex, and the cellular uptake efficiency was analyzed by FACS. The results demonstrated the transfection efficiency of FITC-labeled DNAzymes + Fugene was 56.8\%, FITC-labeled DNAzymes/Lipiodol/Fugene complex was $35.7 \%$ (Fig. 2A). Immunofluorescence also showed VEGDR1 DNAzyme released from Lipiodol (Fig. 2B). These results suggested that addition of Lipiodol in the transfection complex had some effect on transfection efficiency in cells, but still maintained the capacity of transfecting endothelial cells.

Downregulation of VEGFR-1 expression in tumor-bearing rabbits. To examine whether DNAzyme in Fugene/Lipiodol complex delivered via transcatheter into rabbit liver was biologically active, the tumor-bearing rabbits were treated with DNAzyme and control, and the liver cancer samples were collected 8 days post-treatment for RNA extraction for qRT-PCR. As shown in Fig. 3, VEGFR-1 mRNA level was downregulated in the DNAzyme-treated rabbit in comparison with the control. Both Lipiodol and control groups showed some effect of the treatment on VEGFR-1 expression, which may be due to the potential deleterious effect on both tumor and endothelial cells. The data suggested that the use of Lipiodol/Fugene to deliver DNAzyme via a transcatheter is feasible in an in vivo setting.

Effect of transcatheter-delivered VEGFR-1 DNAzyme on tumor vasculature permeability. Next, we investigated the impact of 


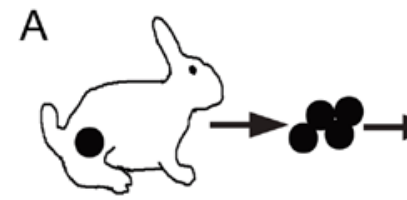
with hind limb
Tumor-bearing
Rabbit Liver

\section{陑}

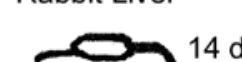

Transcatheter arterial DZ

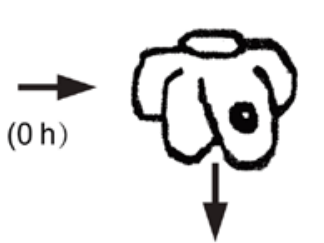

8 days after treatment rabbits were sacrificed
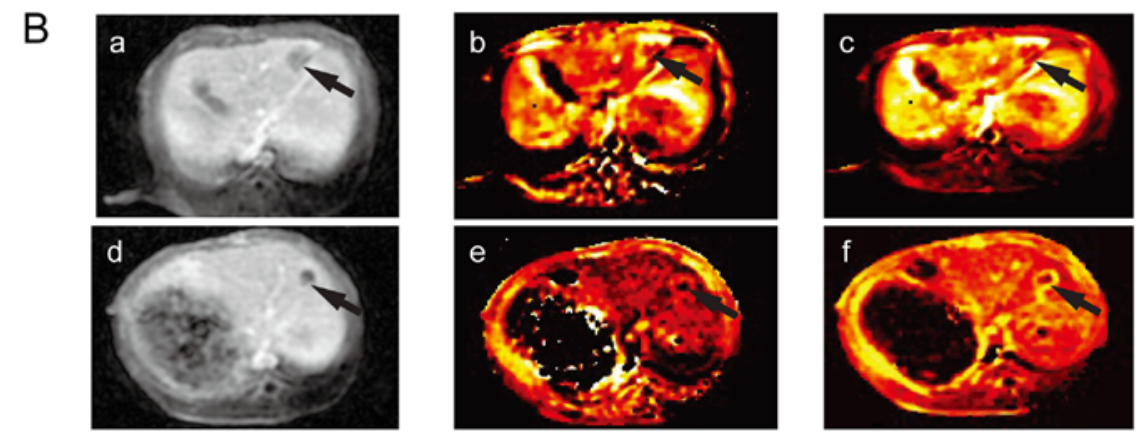

C

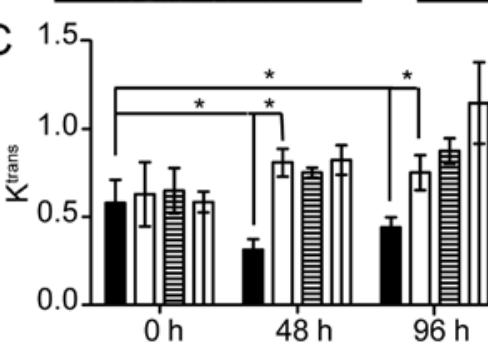
$\mathrm{DZ}+\mathrm{F}+\mathrm{L} 1.5$
DCE-MR

Treatment $(0 \mathrm{~h})$

DCE-MRI after

Treatment (48 h,96h)
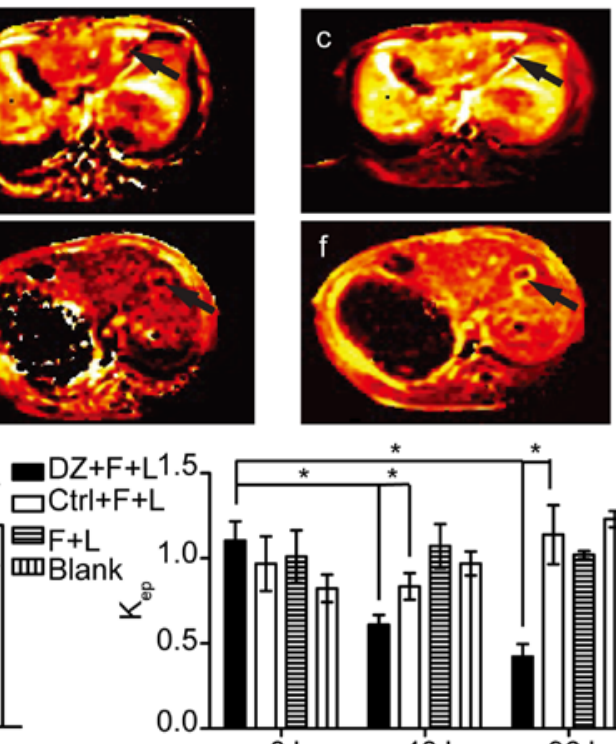

$\mathrm{Oh}$

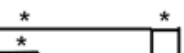

1ำ

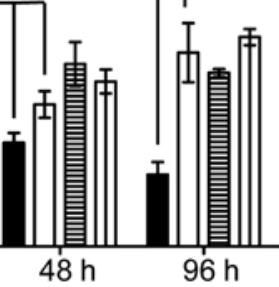

Figure 4. Effect of DZ on tumor angiogenesis assayed by DCE-MRI. (A) Schematic description of the experimental design. (B) DCE-MRI was performed 0 , 48 and $96 \mathrm{~h}$ after DZ treatment. The representative dynamic enhanced MR images were presented for calculation of $\mathrm{K}^{\text {trans }}$ and $\mathrm{K}_{\mathrm{ep}}$ from raw data of DCE-MRI by NordicICE software (v 2.3.14) at $48 \mathrm{~h}$ after treatment. a-c, Images from blank group; d-f, images from treatment group (DZ + F + L). $(\mathrm{C}) \mathrm{K}^{\text {trans }}$ and $\mathrm{K}_{\mathrm{ep}}$ values are expressed as means $\pm \mathrm{SE}$ of three rabbits. ${ }^{*} \mathrm{P}<0.05$ compared with the control group.

the VEGFR-1-targeted DNAzyme on tumor vasculature in tumor-bearing rabbits. DCE-MRI was used as a non-invasive in vivo method to analyze the change of the tumor vasculature. The parameter of $\mathrm{K}^{\text {trans }}$ derived from DCE-MRI reflects the condition of tumor microvascular permeability and has been widely used for evaluation of antitumor drugs. The schedule for treatment and acquiring raw data is shown in Fig. 4A and the $\mathrm{K}^{\text {trans }}$ and $\mathrm{K}_{\mathrm{ep}}$ were calculated based on the images acquired from the animals (Fig. 4B). The results showed that the $\mathrm{K}^{\text {trans }}$ and $\mathrm{K}_{\mathrm{ep}}$ values in DNAzyme-treated group were lower than that in control group after therapy at $48 \mathrm{~h}$ and $96 \mathrm{~h}$ time points $(\mathrm{P}<0.05)$ (Fig. 4C). These results indicated that VEGFR-1targeted DNAzymes could downregulate the VEGFR-1 expression in rabbit liver cancer tissue, leading to change of tumor vasculature and vessel permeability.

\section{Discussion}

DNAzymes offer more advantages than other oligonucleotide agents. For example, DNAzymes, being composed of DNA, are not only easier and less expensive to synthesize, but also much more resistant to degradation in vivo than RNA molecules. A number of DNAzymes targeting c-Jun, LMP-1 and VEGFR-2, are being evaluated in human and animal trials, offering more therapeutic choices for patients (20-22). However, one of the principal barriers to the achievement of the clinical applications of DNAzymes is lack of efficient and safe delivery system. The present study assessed the feasibility of local delivery of the targeted VEGFR-1 DNAzyme via transcatheter in a rabbit liver cancer model. This approach has been clinically used in transcatheter arterial chemoembolization (TACE) for liver cancer treatment. Simulating clinical settings, we replaced chemotherapeutics with DNAzyme in Lipiodol-based embolism. The present study demonstrated that the DNAzyme can be delivered into the liver cancer in the designed schedule and caused disruption of the tumor vasculature, which proved the feasibility of the approach to HCC treatment via molecularly targeted agents in combination with TACE.

Over the past 10 years, DCE-MRI and extracted kinetic parameters have been used as an in vivo cancer imaging tool for the diagnosis, monitoring of treatment effect and evaluation of anticancer drugs. This method has been applied to phase I and II clinical trials of anti-angiogenic drugs and vascular disrupting agents $(23,24)$. In particular, $\mathrm{K}^{\text {trans }}$ is currently recognized as a general marker of tumor blood flow, and has been recommended as a primary endpoint for an anticancer treatment trial by the US National Cancer Institute. $\mathrm{K}^{\text {trans }}$ is a factor that accounts for the complex functions of blood flow, endothelial surface area and endothelial permeability. However, it can also have multiple physiologic interpretations depending on the balance between capillary permeability and blood flow in a tissue. In high-permeability situations (where 
flux across the endothelium is flow limited), $\mathrm{K}^{\text {trans }}$ is equal to the blood plasma flow/unit volume of tissue. Conversely, for low permeability conditions where tracer flux is permeability limited, the $\mathrm{K}^{\text {trans }}$ value is equal to the permeability surface area product/unit volume of tissue. Considering the pathological relevance of VEGFR-1 to angiogenesis, we previously showed that targeting VEGFR-1 by DNAzymes caused significant change of the tumor vasculatures (12). In the present study, the established rabbit HCC model provided a more operable and measurable system to examine the effect of TACE-mediated DNAzyme delivery on tumor vasculatures. The present study showed that VEGFR-1 DNAzymes caused a decline of the $\mathrm{K}^{\text {trans }}$ value for rabbit HCC as demonstrated by DCE-MRI, implying that VEGFR-targeted DNAzymes reduced the vessel leakiness and changed the vascular permeability.

In conclusion, DNAzyme therapeutics can be delivered into HCC via TACE in a much more clinically relevant manner. In vivo, the therapeutic effect of VEGFR-1 targeted DNAzyme can be assessed by DCE-MRI for its impact on tumor vasculature.

\section{Acknowledgements}

The present study was supported by a grant from the Ministry of Science and Technology, China (2013BAI01B07).

\section{References}

1. Stewart BW and Wild CP (eds): The global and regional burden of cancer. World Cancer Report. WHO, pp26-31, 2014.

2. Imai N, Ishigami M, Ishizu Y, Kuzuya T, Honda T, Hayashi K, Hirooka Y and Goto H: Transarterial chemoembolization for hepatocellular carcinoma: A review of techniques. World $\mathrm{J}$ Hepatol 6: 844-850, 2014.

3. Cockman ME, Masson N, Mole DR, Jaakkola P, Chang GW, Clifford SC, Maher ER, Pugh CW, Ratcliffe PJ and Maxwell PH: Hypoxia inducible factor-alpha binding and ubiquitylation by the von Hippel-Lindau tumor suppressor protein. J Biol Chem 275: 25733-25741, 2000.

4. Lim CS, Kiriakidis S, Sandison A, Paleolog EM and Davies AH: Hypoxia-inducible factor pathway and diseases of the vascular wall. J Vasc Surg 58: 219-230, 2013.

5. Otrock ZK, Makarem JA and Shamseddine AI: Vascular endothelial growth factor family of ligands and receptors: Review. Blood Cells Mol Dis 38: 258-268, 2007.

6. Dias S, Hattori K, Heissig B, Zhu Z, Wu Y, Witte L, Hicklin DJ, Tateno M, Bohlen P, Moore MA, et al: Inhibition of both paracrine and autocrine VEGF/VEGFR-2 signaling pathways is essential to induce long-term remission of xenotransplanted human leukemias. Proc Natl Acad Sci USA 98: 10857-10862, 2001.

7. Villegas G, Lange-Sperandio B and Tufro A: Autocrine and paracrine functions of vascular endothelial growth factor (VEGF) in renal tubular epithelial cells. Kidney Int 67: 449-457, 2005.

8. Sison K, Eremina V, Baelde H, Min W, Hirashima M, Fantus IG and Quaggin SE: Glomerular structure and function require paracrine, not autocrine, VEGF-VEGFR-2 signaling. J Am Soc Nephrol 21: 1691-1701, 2010.
9. Peng S, Wang Y, Peng H, Chen D, Shen S, Peng B, Chen M, Lencioni R and Kuang M: Autocrine vascular endothelial growth factor signaling promotes cell proliferation and modulates sorafenib treatment efficacy in hepatocellular carcinoma. Hepatology 60: 1264-1277, 2014.

10. Marchand GS, Noiseux N, Tanguay JF and Sirois MG: Blockade of in vivo VEGF-mediated angiogenesis by antisense gene therapy: Role of Flk-1 and Flt-1 receptors. Am J Physiol Heart Circ Physiol 282: H194-H204, 2002.

11. Pavco PA, Bouhana KS, Gallegos AM, Agrawal A, Blanchard KS, Grimm SL, Jensen KL, Andrews LE, Wincott FE, Pitot PA, et al: Antitumor and antimetastatic activity of ribozymes targeting the messenger RNA of vascular endothelial growth factor receptors. Clin Cancer Res 6: 2094-2103, 2000.

12. Shen L, Zhou Q, Wang Y, Liao W, Chen Y, Xu Z, Yang L and Sun LQ: Antiangiogenic and antitumoral effects mediated by a vascular endothelial growth factor receptor 1 (VEGFR-1)-targeted DNAzyme. Mol Med 19: 377-386, 2013.

13. Ivy SP, Wick JY and Kaufman BM: An overview of small-molecule inhibitors of VEGFR signaling. Nat Rev Clin Oncol 6: 569-579, 2009.

14. Sun LQ, Cairns MJ, Saravolac EG, Baker A and Gerlach WL: Catalytic nucleic acids: From lab to applications. Pharmacol Rev 52: 325-347, 2000.

15. Schubert S, Gül DC, Grunert HP, Zeichhardt H, Erdmann VA and Kurreck J: RNA cleaving '10-23' DNAzymes with enhanced stability and activity. Nucleic Acids Res 31: 5982-5992, 2003

16. Chen X, Xiao E, Shu D, Yang C, Liang B, He Z and Bian D: Evaluating the therapeutic effect of hepatocellular carcinoma treated with transcatheter arterial chemoembolization by magnetic resonance perfusion imaging. Eur J Gastroenterol Hepatol 26: 109-113, 2014.

17. Prat F, Centarti M, Sibille A, Abou el Fadil FA, Henry L, Chapelon JY and Cathignol D: Extracorporeal high-intensity focused ultrasound for VX2 liver tumors in the rabbit. Hepatology 21: 832-836, 1995.

18. Okada M, Kudo S, Miyazaki O, Saino T, Ekimoto H, Iguchi H, Hirano S, Kuboki H, Kadosawa H and Takeuchi T: Antitumoral efficacy and pharmacokinetic properties of pirarubicin upon hepatic intra-arterial injection in the rabbit $\mathrm{V} \times 2$ tumour model. Br J Cancer 71: 518-524, 1995.

19. Ramirez LH, Orlowski S, An D, Bindoula G, Dzodic R, Ardouin P, Bognel C, Belehradek J Jr, Munck JN and Mir LM: Electrochemotherapy on liver tumours in rabbits. Br J Cancer 77: 2104-2111, 1998

20. Zhang G, Dass CR, Sumithran E, Di Girolamo N, Sun LQ and Khachigian LM: Effect of deoxyribozymes targeting c-Jun on solid tumor growth and angiogenesis in rodents. J Natl Cancer Inst 96: 683-696, 2004.

21. Yang L, Lu Z, Ma X, Cao Y and Sun LQ: A therapeutic approach to nasopharyngeal carcinomas by DNAzymes targeting EBV LMP-1 gene. Molecules 15: 6127-6139, 2010.

22. Zhang L, Gasper WJ, Stass SA, Ioffe OB, Davis MA and Mixson AJ: Angiogenic inhibition mediated by a DNAzyme that targets vascular endothelial growth factor receptor 2. Cancer Res 62: 5463-5469, 2002.

23. Folkman J: Tumor angiogenesis: Therapeutic implications. $\mathrm{N}$ Engl J Med 285: 1182-1186, 1971.

24. Raskopf E, Vogt A, Sauerbruch T and Schmitz V: siRNA targeting VEGF inhibits hepatocellular carcinoma growth and tumor angiogenesis in vivo. J Hepatol 49: 977-984, 2008. 